\title{
Highly Compressive and Stretchable Poly(Ethylene Glycol) Based Hydrogels Synthesised Using pH-Responsive Nanogels Without Free-Radical Chemistry
} DOI:

10.1039/C9NR01535C

\section{Document Version \\ Accepted author manuscript}

Link to publication record in Manchester Research Explorer

Citation for published version (APA):

Saunders, B. (2019). Highly Compressive and Stretchable Poly(Ethylene Glycol) Based Hydrogels Synthesised Using pH-Responsive Nanogels Without Free-Radical Chemistry. Nanoscale, 11, 7921.

https://doi.org/10.1039/C9NR01535C

\section{Published in:}

Nanoscale

\section{Citing this paper}

Please note that where the full-text provided on Manchester Research Explorer is the Author Accepted Manuscript or Proof version this may differ from the final Published version. If citing, it is advised that you check and use the publisher's definitive version.

\section{General rights}

Copyright and moral rights for the publications made accessible in the Research Explorer are retained by the authors and/or other copyright owners and it is a condition of accessing publications that users recognise and abide by the legal requirements associated with these rights.

\section{Takedown policy}

If you believe that this document breaches copyright please refer to the University of Manchester's Takedown Procedures [http://man.ac.uk/04Y6Bo] or contact uml.scholarlycommunications@manchester.ac.uk providing relevant details, so we can investigate your claim.

\section{OPEN ACCESS}



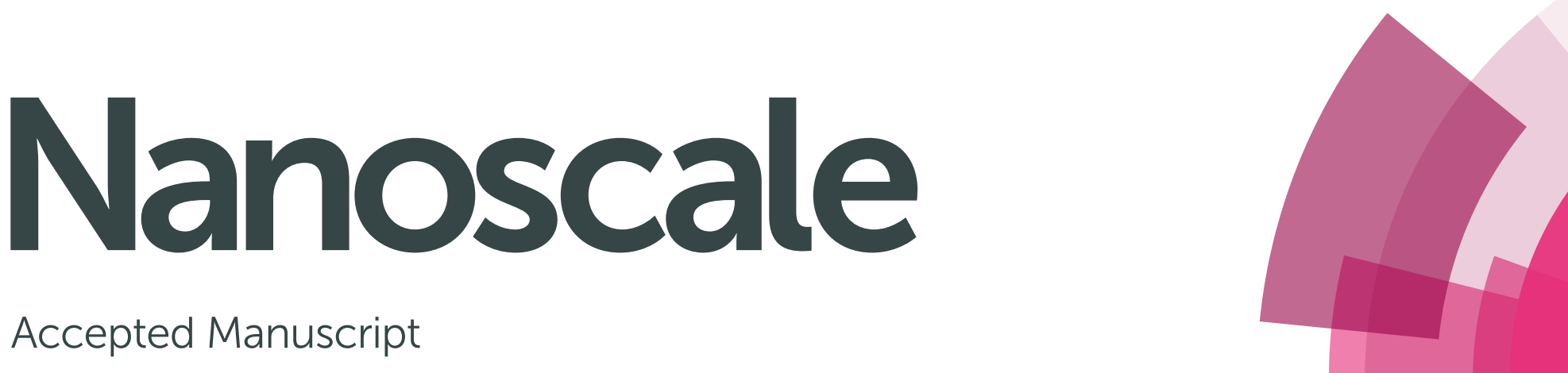

\section{Accepted Manuscript}

This article can be cited before page numbers have been issued, to do this please use: N. T. Nguyen, A. H. Milani, J. Jennings, D. J. Adlam, T. Freemont, J. A. Hoyland and B. R. Saunders, Nanoscale, 2019, DOI:
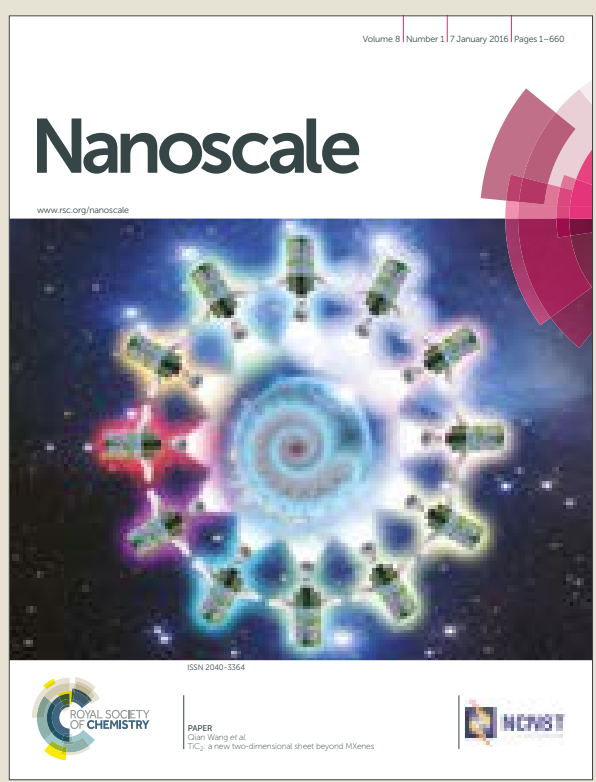

This is an Accepted Manuscript, which has been through the Royal Society of Chemistry peer review process and has been accepted for publication.

Accepted Manuscripts are published online shortly after acceptance, before technical editing, formatting and proof reading. Using this free service, authors can make their results available to the community, in citable form, before we publish the edited article. We will replace this Accepted Manuscript with the edited and formatted Advance Article as soon as it is available.

You can find more information about Accepted Manuscripts in the author guidelines.

Please note that technical editing may introduce minor changes to the text and/or graphics, which may alter content. The journal's standard Terms \& Conditions and the ethical guidelines, outlined in our author and reviewer resource centre, still apply. In no event shall the Royal Society of Chemistry be held responsible for any errors or omissions in this Accepted Manuscript or any consequences arising from the use of any information it contains. 


\title{
Highly Compressive and Stretchable Poly(Ethylene Glycol) Based Hydrogels Synthesised Using pH-Responsive Nanogels Without Free-Radical Chemistry.
}

Received 00th January 20xx, Accepted 00th January 20xx

DOI: $10.1039 / \times 0 \times x 00000 x$

www.rsc.org/

\author{
Nam T. Nguyen ${ }^{\mathrm{a},{ }^{*}}$, Amir H. Milani ${ }^{\mathrm{a}}$, James Jennings ${ }^{\mathrm{b}}$, Daman J. Adlam ${ }^{\mathrm{c}}$, Anthony J. Freemont ${ }^{\mathrm{c}}$, \\ Judith A. Hoyland ${ }^{c, d}$ and Brian R. Saunders. ${ }^{a}$
}

\begin{abstract}
Poly(ethylene glycol) (PEG) based hydrogels are amongst the most studied synthetic hydrogels. However, reports on PEGbased hydrogels with high mechanical strength are limited. Herein, a class of novel, well-defined PEG-based nanocomposite hydrogels with tunable mechanical strength are synthesised via ring-opening reactions of diglycidyl ethers with carboxylate ions. The $\mathrm{pH}$ responsive crosslinked polyacid nanogels (NG) in the dispersed phase act as high functionality crosslinkers which covalently bond to the poly(ethylene glycol) diglycidyl ethers (PEGDGE) as the continuous matrix. A series of NG-xPEG-y-z gels are prepared where $x, y$ and $z$ are concentrations of NGs, PEGDGE and the PEGDGE molecular weight, respectively. The hydrogel compositions and nano-structural homogeneity of the NGs have strong impact on the enhancement of mechanical properties which enables property tuning. Based on this design, a highly compressive PEG based nanocomposite hydrogel (NG-13-PEG-20-6000) exhibits a compressive stress of $24.2 \mathrm{MPa}$, compressive fracture strain greater than $98 \%$ and a fracture energy density as high as $1.88 \mathrm{MJ} \mathrm{m}^{-3}$. The tensile fracture strain is $230 \%$. This is amongst one of the most compressive PEG-based hydrogels reported to-date. Our chemically crosslinked gels are resilient and show highly recoverable dissipative energy. The cytotoxicity test shows that human nucleus pulposus (NP) cells remained viable after 8 days of culture time. The overall results highlight their potential for applications as replacements for intervertebral discs or articular cartilages.
\end{abstract}

\section{Introduction}

Synthetic hydrogels have attracted great attention in the past few decades as a new class of soft materials for many biomedical applications including tissue engineering, drug/gene delivery and injectable/implant devices. ${ }^{1-7}$ They have advantages over their natural counterparts such as the ability to be tailored with the desired mechanical properties for applications. In particular, poly(ethylene glycol) (PEG) based hydrogels are amongst the most studied synthetic hydrogels. Due to the non-adhesive nature of PEG chains, PEG hydrogels typically exhibit minimal or no intrinsic biological activity. Extensive reviews on PEG hydrogels can be found in the literature. ${ }^{6-9}$ Many synthetic pathways have been employed for the synthesis of PEG-based hydrogels including free-radical

\footnotetext{
a. School of Materials, University of Manchester, Manchester, M13 9PL, United Kingdom

b. Department of Chemistry, University of Sheffield, Brook Hill, Sheffield, South Yorkshire S3 $7 H F$, United Kingdom

Division of Cell Matrix Biology and Regenerative Medicine, Faculty of Biology, Medicine and Health, University of Manchester, Oxford Road, Manchester, M13 SPT, United Kingdom

d. NIHR Manchester Biomedical Research Centre, Manchester University NHS foundation Trust, Manchester Academic Health Science Centre, M13 9WL, United Kingdom

tElectronic Supplementary Information (ESI) available: [details of any supplementary information available should be included here]. See DOI: $10.1039 / x 0 x x 00000 x$
}

polymerisation of PEG di-/multi-acrylates, ${ }^{10-14}$ condensation, ${ }^{15}$ Michael-type addition, ${ }^{16-18}$ Click chemistry ${ }^{19-21}$ and ring-opening reaction. 22-34 The use of a commercially available poly(ethylene glycol) diglycidyl ether (PEGDGE) for ring-opening reaction has been studied for the construction of 3D-networks. ${ }^{22-34}$ One big advantage of PEGDGE is that the ring-opening crosslinking can be carried out directly on the diglycidyl ether (or diepoxide) functional groups without time and cost consuming synthetic steps. In most cases, the PEGDGE's epoxides were ring-opened using amine, 22-24 hydroxyl, ${ }^{25-30}$ phenolic $^{31-33}$ and thiol ${ }^{34}$ functional groups. Furthermore, it is well-known that the ringopening reaction of epoxides can occur with carboxylic acid. ${ }^{35-}$ 44 To date, however, there are no reports on crosslinking between polyacid molecules and PEGDGE for the formation of hydrogels.

High mechanical strength hydrogels are desired for many hydrogels, especially for applications as replacements of degenerated human cartilage or intervertebral discs. ${ }^{45}$ One approach to enhance the mechanical properties is to use double network (DN) hydrogels formed by combining two different polymers. ${ }^{46}$ Recent studies have also reported hydrogels with polymerisation-formed nanostructures provide enhanced mechanical properties. ${ }^{47-49}$ Another approach is to construct the micro/nano composite hydrogels in which a dispersed phase of micro/nano particles are distributed in a matrix by physical or chemical crosslinking. ${ }^{50-69}$ Reports on hydrogels with excellent mechanical strength are mostly based on $\mathrm{DN}^{46,70}$, 
hybrid $D^{71}, 72$ and micro/nano particle reinforced $D N$ hydrogels. ${ }^{52,56,61,65,73}$

Our group introduced the formation of a double crosslinked hydrogel by free-radical crosslinking of vinyl bonds between the glycidyl methacrylate functionalised $\mathrm{pH}$-responsive micro/nanogels. ${ }^{39-44}$ The $\mathrm{pH}$-responsive ability of hydrogels is mostly the result of $\mathrm{pH}$-dependent swelling of the particles. However, because of the random reactions between vinyl groups, the hydrogels sometimes exhibited mechanical weakness. $^{50}$ Here, we propose a novel, PEG-based nanocomposite hydrogel with high mechanical strength prepared via a non-free radical approach using ring-opening reactions of the diglycidyl ether by carboxylate ions located on polyacid nanogels (NGs). The pH responsive singly crosslinked $\mathrm{NGs}^{42,} 43$ act as a high functionality crosslinker which is covalently bonded to the continuous matrix of PEGDGE. The effect of the composition, such as PEGDGE molecular weight, the amount of the NG and PEGDGE on the hydrogel mechanical properties were investigated in detail. We show that an optimized formulation produced hydrogels with remarkable compressive strengths.

\section{Experimental}

\section{Materials.}

Methyl methacrylate (MMA, Sigma-Aldrich, 99\%), methacrylic acid (MAA, Sigma-Aldrich, 99\%), ethyleneglycol dimethacrylate (EGDMA, Sigma-Aldrich, 98\%), ammonium persulfate (APS, Sigma-Aldrich, 98\%), sodium dodecyl sulfate (SDS, SigmaAldrich, 98.5\%), poly(ethylene glycol) diglycidyl ether (PEGDGE, Sigma-Aldrich, 99\%), $\mathrm{NaOH}$ (Sigma-Aldrich, 99\%) were used as received. All water was of ultra-high purity and was deionized and doubly distilled.

\section{Synthesis of singly crosslinked nanogel particles containing MMA, MAA, EGDMA.}

The singly crosslinked NGs consisting of MMA, MAA and EGDMA (poly(MMA-co-MAA-co-EDGMA)) were synthesised via semi-continuous emulsion polymerisation as reported elsewhere ${ }^{43}$. Briefly, SDS $(1.20 \mathrm{~g}, 4.0 \mathrm{mmol})$ was dissolved in water $(240 \mathrm{ml})$ and the solution purged with nitrogen for 30 $\mathrm{min}$. Then APS $(0.20 \mathrm{~g}, 0.90 \mathrm{mmol})$ in water $(2.0 \mathrm{ml})$ was added. The semi-continuous emulsion polymerisation was conducted at $80^{\circ} \mathrm{C}$. A comonomer solution containing MMA (42.0 g, 0.42 mol), MAA (10.0 g, $0.12 \mathrm{~mol})$ and EGDMA ( $1.10 \mathrm{~g}, 5.0 \mathrm{mmol}$ ) was added at a feed rate of $0.40 \mathrm{~mL} / \mathrm{min}$. The reaction was left to stir for $1 \mathrm{~h}$ after the feed finished and then the reaction cooled to room temperature. The dispersion purified by dialysis against water with stirring for 7 days.

\section{Synthesis of PEG-based nanocomposite hydrogel using NG as a multifunctional crosslinker.}

Prior to the fabrication of the nanocomposite hydrogel using PEGDGE and NG, concentrated NG dispersion was prepared as follows: NG dispersion after intensive dialysis was filtered via $0.2 \mu \mathrm{m}$ Cellulose membrane. The filtered dispersion was concentrated by rotary evaporation at room temperature

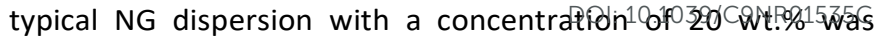
prepared from higher concentrated dispersion by diluting with both water and $\mathrm{NaOH}(2.0 \mathrm{M})$ solution. In a typical preparation, PEGDGE $\left(0.3 \mathrm{~g}\right.$ ) with molecular weight of $6000 \mathrm{gmol}^{-1}$ (denoted PEGDGE6000) was weighed and slowly added to NG dispersion (1.0g of 20 wt.\%) at pH 6.4 while the vial was subjected to continuous vortex. An additional quantity of water $(200 \mu \mathrm{L})$ was then added. Upon mixing, the final $\mathrm{pH}$ was 7.0. The masses used to prepare the gels and their compositions are summarised in Supporting Information Tables S1. The mixtures were then transferred into appropriate moulds and cured for $24-48 \mathrm{~h}$ in an oven at $37^{\circ} \mathrm{C}$.

\section{Swelling studies.}

The volume swelling ratio of the gels was measured using a gravimetric method. Samples were placed in phosphate buffer solution at different $\mathrm{pH}$ values $(0.1 \mathrm{M})$. Each sample was weighed once a day and left in a fresh buffer. This process was repeated for 5 days. The volume swelling ratio $(Q)$ for the gels was calculated using:

$$
Q=\rho_{p}\left(\frac{Q_{(m)}}{\rho_{s}}+\frac{1}{\rho_{p}}\right)-\frac{\rho_{p}}{\rho_{s}}
$$

where $Q_{(m)}$ is the mass swelling ratio. The $\rho_{s}$ and $\rho_{p}$ are the densities of the solvent (water) and polymer, respectively. These were taken as 1.0 and $1.2 \mathrm{~g} \mathrm{ml}^{-1} .^{74}$

\section{Cytotoxicity tests.}

Human nucleus pulposus (NP) cells were derived from intervertebral disc samples obtained with informed patient consent under ethical approval from the research ethics committee (London - Brighton \& Sussex. Ref. No: 17/LO/1408 'Improving the understanding of intervertebral disc degeneration, diagnosis and treatment'). NP cells were cultured in Dulbecco's modified Eagle's medium (DMEM) supplemented with antibiotic/antimycotic (Sigma-Aldrich, UK), Glutamax and $10 \%$ fetal bovine serum (Thermo Fisher Scientific, UK) within a humidified $5 \% \mathrm{CO}_{2}$ incubator at $37^{\circ} \mathrm{C}$. Cells were harvested by trypsinisation and seeded at a density of $3 \times 10^{4}$ cells per well onto $13 \mathrm{~mm}$ sterile glass coverslips in 24 well culture plates. After overnight incubation, NP cell culture media was replenished and toroid shaped gels, sterilized in $70 \%$ ethanol for 10 minutes and rehydrated with several changes of sterile phosphate buffered saline (PBS) over a $72 \mathrm{~h}$ period, were introduced into the appropriate wells. Control cultures received an equal volume of PBS. Cells were cultured up to eight days and live/dead assays (Thermo Fisher Scientific, UK) were performed at 1, 4 and 8 day according to the manufacturer's instructions. Images were obtained with an Olympus BX51 fluorescence microscope and an EVOS XL Core cell imaging system.

\section{Physical Measurements.}

Potentiometric titration data were obtained using a Mettler Toledo titrator using $\mathrm{NaOH}(1.0 \mathrm{M})$ as the titrant. Typically, a NG dispersion ( $40 \mathrm{~mL}$ and $0.5 \mathrm{wt} . \%$ ) was prepared in aqueous $\mathrm{NaCl}$ 
solution (0.050 M). Dynamic light scattering (DLS) and zeta potential data were obtained using a Malvern Zetasizer NanoZS instrument. A Nicolet 5700 (Thermo Electron Corporation) spectrophotometer was used for Fourier transform infrared (FTIR) spectroscopy measurements.

\section{Transmission Electron Microscopy.}

Holey carbon-coated copper grids were subjected to plasma glow-discharge for $40 \mathrm{~s}$ to create a hydrophilic surface. The NG dispersion (1.0 wt.\%, $20 \mu \mathrm{L}$ ) was placed onto a freshly prepared grid for $1 \mathrm{~min}$ and then aqueous uranyl formate (0.75 wt.\%, 20 $\mu \mathrm{L}$ ) added for $30 \mathrm{~s}$. Excess stain was removed by deionised water. Each grid was then carefully dried at atmospheric condition. Imaging was performed at $100 \mathrm{kV}$ using a Phillips CM100 instrument equipped with a Gatan 1k CCD camera. The number-average diameters were determined using Image-J (NIH) software.

\section{Scanning Electron Microscopy.}

The hydrogels were rapidly frozen in liquid nitrogen and then freeze-dried overnight. The samples were mounted on Al slides using carbon tape and coated with Au. The morphology of the hydrogels was examined using a Philips FEGSEM instrument at an accelerating voltage of $12 \mathrm{kV}$.

\section{Dynamic rheology measurements.}

All the mechanical tests were conducted on the as-prepared hydrogels unless otherwise stated. All rheological studies were performed on a TA Instruments AR200 rheometer. A $20 \mathrm{~mm}$ diameter plate geometry was used and the gap was set to 2500 $\mu \mathrm{m}$. All measurements were conducted at $25^{\circ} \mathrm{C}$. Frequency sweeps were performed at $1.0 \%$ strain and at oscillation frequencies of $0.1-200 \mathrm{rad} \mathrm{s}^{-1}$.

\section{Compressive properties.}

All uniaxial compression tests were performed using an Instron 3344. The gels were prepared in PTFE cylindrical moulds with a typical dimension of $11.50 \mathrm{~mm} \times 11.00 \mathrm{~mm}$ height $\times$ diameter. The specimens were compressed between two plates with a strain rate of $2.0 \mathrm{~mm} / \mathrm{min}$ until fracture or until the maximum load value was reached. The engineering stress was used in these studies. The cyclic compression tests were performed using a compression rate of $5.0 \mathrm{~mm} / \mathrm{min}$ for 20 cycles.

\section{Tensile properties.}

For tensile tests, the gels were prepared as dumbbell shape using PTPE moulds with a typical thickness of $3 \mathrm{~mm}$, sealed by two glass slides. The dimensions of the mould midsection was $5 \mathrm{~mm} \times 32 \mathrm{~mm}$ width $\times$ length. The specimens were clamped and stretched with a strain rate of $1.0 \mathrm{~mm} / \mathrm{min}$ until fracture.

\section{Small-angle X-ray Scattering.}

SAXS measurements were performed in the SMALL laboratory at the University of Sheffield on a Xeuss 2.0 (Xenocs, Sassenage, France) at room temperature. X-rays (1.341 $\AA$ ) were generated by a liquid gallium MetalJet X-ray source (Excillum, Kista, Sweden), and the beam size was collimated through a pair of scatterless slits (1.2 and $0.8 \mathrm{~mm})$. Two-dimensignale XRP

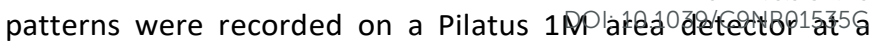
sample-to-detector distance of $1.225 \mathrm{~m}$ (calibrated using a silver behenate standard). Two-dimensional SAXS patterns were azimuthally integrated using the Foxtrot software package to obtain 1D scattering profiles. The scattering vector $(q)$ values of structure factor maxima $\left(q_{\max }\right)$ were obtained by Lorentzian peaks in Igor Pro to a Kratky plot ( $2 \theta$ vs. $1 \mathrm{Iq}^{2}$ to correct for instrument geometry $\left.{ }^{75},{ }^{76}\right)$ and nanogel centre-to-centre distances $(D)$ were calculated from the relationship $D=2 \pi / q_{\text {max }}$. For the pre-gel sample, a sample of PEG6000 solution was used to subtract as a background prior to fitting, whereas data from all hydrogels were fitted as collected (See Figure S6, ESIt for examples of fitting).

\section{Results and discussion}

The PEG-based nanocomposite hydrogels were prepared in one step by simply mixing NG and commercially available PEGDGE without any further synthetic modifications (see Scheme 1).

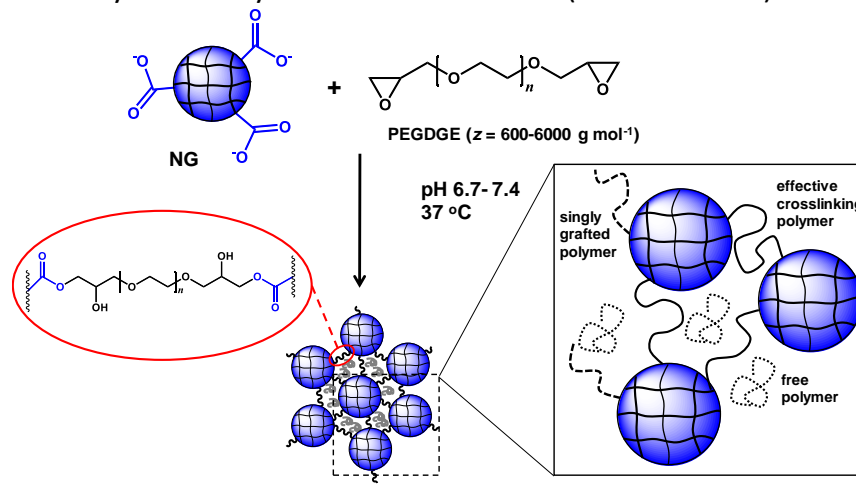

NG crosslinked PEG gel (NG-x-PEG-y-z )

Scheme 1. Depiction of the preparation of NG-x-PEG-y-z gels, where $x$ and $y$ are the final concentrations in the formulation of $N G$ and PEGDGE, respectively. $Z$ is the molecular weight of PEGDGE.

A large scale synthesis typically resulted in approximately 1 litre of NG solution ( $3.5 \mathrm{wt} . \%$ ) which demonstrates the feasibility for large-scale NG preparation. At $\mathrm{pH}$ higher than 6.0, the carboxylic acids can act as nucleophiles to ring-open the epoxides on both ends of PEGDGE to form covalent crosslinking (See Scheme 1). ${ }^{35-37}$ The $\mathrm{pH}$ range at which network formation occurred corresponds to the physiological range.

We improved the previously reported synthesis of NG particles consisting of poly(MMA-co-MAA-co-EDGMA), ${ }^{42,} 43$ by following the particle growth so that smaller size NG could be synthesised. Compared to their larger size counterparts, smaller size NGs could be advantageous as high functionality crosslinkers because their higher surface area could provide higher crosslink density. In addition, higher particle concentration has been reported as a critical parameter for the enhancement of hydrogel mechanical properties, as a result of better stress distribution. ${ }^{77}$ The NG had a number-average diameter of $17 \mathrm{~nm}$ by TEM (Figure 1A). Potentiometric titration data (Figure $\mathrm{S} 1, \mathrm{ESI}+$ ) showed that the $\mathrm{p} K_{a}$ of the NG is 6.9 and the particles contained 35.9 wt.\% of MAA. The average particle sizes is smaller than previously reported 43 and the NG 
contained a higher concentration of MAA. DLS data (Figure 1B) and zeta potential measurements (Figure S1, ESI+) clearly show the $\mathrm{pH}$-responsiveness where the NG can swell from $24 \mathrm{~nm}$ at $\mathrm{pH} 5.8$ to up to $50 \mathrm{~nm}$ at $\mathrm{pH} 9.8$. The zeta potentials showed that they were negatively charged at all $\mathrm{pH}$ values studied. Figure S1C and Figure S1D (ESI $\left.{ }^{+}\right)$indicate a narrow size distribution with polydispersity index (PDI) values in the range of $0.06-0.13$ at different $\mathrm{pH}$ conditions.

We found that the presence of both the NGs and PEGDGE were critical for a successful synthesis of the nanocomposite hydrogel. Time-dependent inverted vial tests suggested that it was not possible to form any self-standing gels without either the NGs or PEGDGE (Figure $1 \mathrm{C}$ and Figure S2, ESI + ). FT-IR spectroscopy (Figure 1D) showed that the gel formation is associated with the significant depletion of the $914 \mathrm{~cm}^{-1}$ absorption peak, which is a signature of epoxide group conversion. 78
(A)

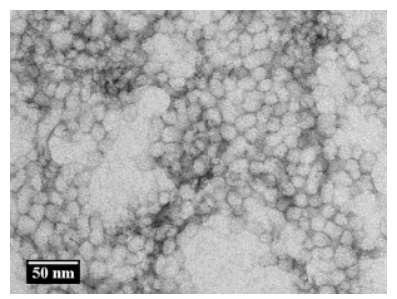

(C)

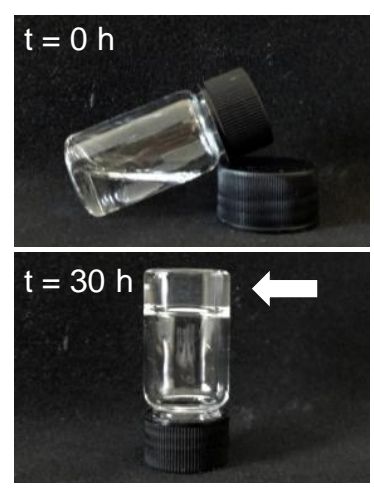

(B)

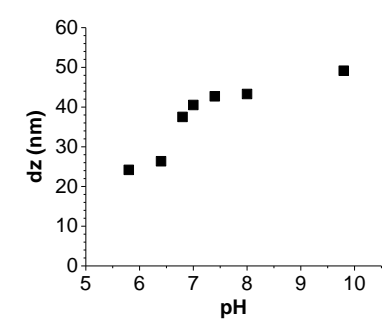

(D)

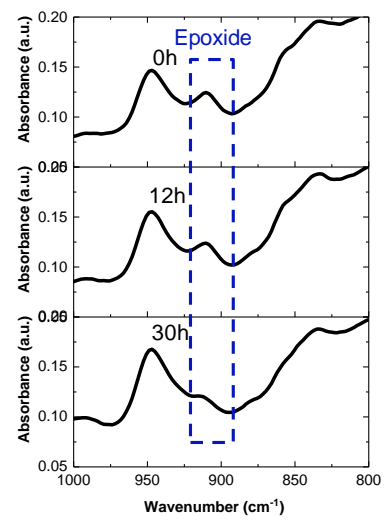

Figure 1. Characterization of poly(MMA-co-MAA-co-EDGMA) NG. (A) TEM image of the NGs. (B) Variation of NG z-average diameter $\left(\mathrm{d}_{\mathrm{z}}\right)$ with $\mathrm{pH}$ as measured by DLS. (C) Digital photographs of the inverted vials showing formation of gel from a dispersion of NG and PEGDGE600; the arrow shows the gel. (D) FTIR spectroscopy study on the kinetics of formation of NG-9-PEG-45-600 nanocomposite hydrogel at time $t=0,12$ and $30 \mathrm{~h}$. The rectangle highlights the epoxide band.

As seen in Figure S2, ESIt, this peak remained largely unchanged when there was only PEGDGE600 present. In contrast the band decreased in the presence of both the NG and PEGDGE (see Figure 1D). Furthermore, suitable $\mathrm{pH}$ conditions were also found to be important to ensure the NG dispersion stability in the presence of PEGDGE. Introduction of PEG could cause the aggregation and flocculation of the NG dispersion due to depletion flocculation. ${ }^{79}$ We found that at $\mathrm{pH} \geq 6.0$ the NG stability was maintained and was not affected significantly by the mass ratio of NG to PEGDGE. Prior to the ring-opening

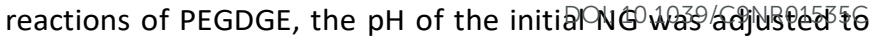
6.4 using $\mathrm{NaOH}$ solution.

An advantage of our approach to gel formation is that PEGDGE with different molecular weights (MW) can be used to prepare hydrogels with targeted mechanical properties. Oscillatory shear rheology were used to initially investigate the effect of PEGDGE molecular weights on the mechanical properties of the NG-9-PEG-45-z gels ( $z=600-6000 \mathrm{~g} \mathrm{~mol}^{-1}$ ). For this gel series, the masses of NG and PEGDGE are kept constant. Therefore, the concentrations of NG and PEGDGE remained constant at $\mathrm{x}=9 \mathrm{wt} . \%$ and $\mathrm{y}=45 \mathrm{wt} . \%$, respectively. Images of several gels are shown in Figure 2A. Rheological properties of the nanocomposite hydrogels are dependent on PEGDGE MW. Frequency-sweep oscillatory rheology (Figure 2 and Figure $\mathrm{S} 3, \mathrm{ESI}+$ ) demonstrated that the elastic (storage) modulus $\left(G^{\prime}\right)$ of all gels are much higher than the viscous (loss) modulus $\left(G^{\prime \prime}\right)$. The frequency independence of $G^{\prime}$ coupled to the relatively low $G$ " values is strong evidence for highly elastic gels without significant viscous dissipation. ${ }^{80}$ Furthermore, as seen in Figure 2D, the $G^{\prime}$ value decrease sharply by increasing the $\mathrm{MW}$ from 600 to $6000 \mathrm{~g} \mathrm{~mol}^{-1}$ suggesting a decrease in numberdensity of elastically effective chains.
(A)

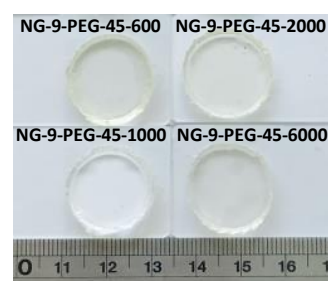

(C)

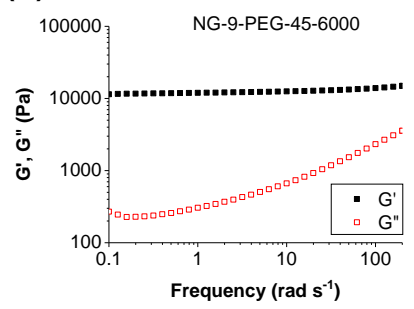

(B)

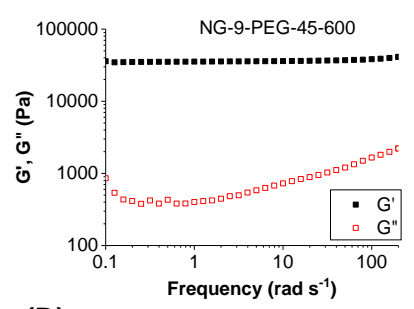

(D)

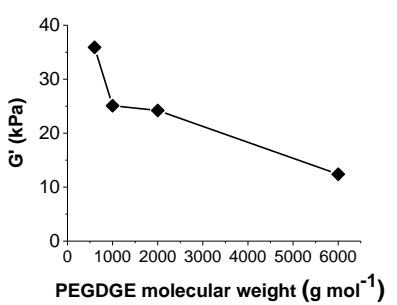

Figure 2. (A) Digital photographs of several self-supporting NG-9-PEG-45-z ( $z=600-6000$ $\mathrm{g} \mathrm{mol}-1$ ) gels. Frequency sweep rheology data for (B) NG-9-PEG-45-600 gel and (C) NG9-PEG-45-6000 gel. (D) The variation of the storage modulus measured at $1 \mathrm{rad} \mathrm{s}^{-1}$ with PEGDGE molecular weight.

The compression studies investigated the effect of $\mathrm{MW}$ on the NG-9-PEG-45-z ( $\left.z=600-6000 \mathrm{~g} \mathrm{~mol}^{-1}\right)$ gel mechanical properties (Figure $3 \mathrm{~A}$ ). The compressive elastic moduli were calculated within the first $10 \%$ strain and had values of $10-200$ $\mathrm{KPa}$ (Table S1, ESI + ). These values are in the region of many hydrogel systems as soft matter for biological applications ${ }^{81}$ and many human soft tissues. ${ }^{82,83}$ They decreased significantly from NG-9-PEG-45-600 to NG-9-PEG-45-1000 then more gradually to NG-9-PEG-45-6000 (Figure 3B). Moreover, fracture strain versus PEGDGE MW showed an opposite trend with a strong, linear dependence on the PEGDGE MW from as low as $27 \%$ up to 96 
\%. In particular, NG-9-PEG-45-6000 gel had the highest compressive stress (4.1 $\mathrm{MPa}$ ) and fracture strain (92\%).

At $\mathrm{pH}>6.0$, the $\mathrm{NGs}$ are well dispersed in aqueous media which enabled uniform dispersion of the NGs within the PEGDGE matrix during gel synthesis. Effective crosslinking occurs when neighbouring NGs connect PEGDGE chains. Polymer chains can also exist as singly grafted or free polymers as a result of incomplete crosslinking (see Scheme 1). Varying the MWs at constant masses of both PEGDGE and NGs ultimately changes the concentration of available epoxide groups and the initial carboxylate to epoxide molar ratio of [COOH]/[Epoxide] (see Table S1, ESI+). For most gels, as the NGs are spherical in shape some of the carboxylic groups in the inner core of the particles may not react. The actual number of reacting carboxylate groups in the NGs may be less than that of epoxide groups. This implies the existence of the free non-
(A)

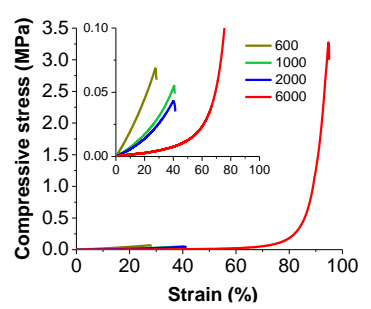

(C)

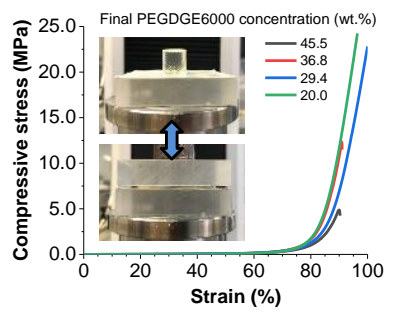

(B)

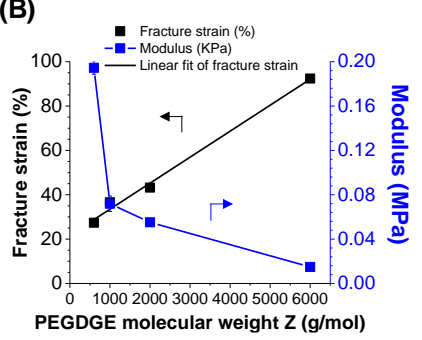

(D)

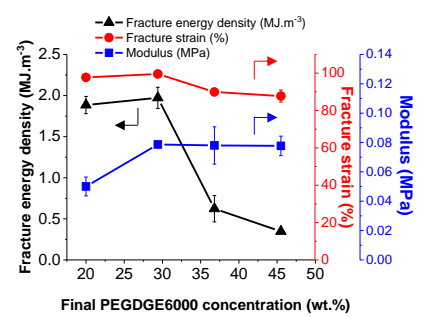

crosslinked PEGDGE and singly grafted polymers embedded in the hydrogel network. ${ }^{84}$

Figure 3. (A) Compressive stress versus strain data for the NG-9-PEG-45-z gels $(z=600-6000 \mathrm{~g} \mathrm{~mol}-1)$. The legend shows the $z$ values. (B) Variation of fracture strain and modulus for the gels with different $z$ values. (C) Compressive stress vs. strain data for NG-x-PEG-y-6000 gels with different final PEGDGE6000 concentration ( $x=9.1-13.3 \mathrm{wt} . \%$ and $y=45.5-20.0 \mathrm{wt} . \%$, respectively). Inset are images of the nanocomposite NG-13-PEG-20-6000 gel for compression test before and after loading. The gel did not break and recovered its original dimensions. (D) Variation of fracture energy density, fracture strain and modulus versus final PEGDGE6000 concentration.

We propose that the crosslinking process became less effective due to shielding/entrapment of the reactive groups by free and singly grafted polymers. This effect was likely less significant when using PEG chains of shorter chain lengths. ${ }^{52,} 81$ At lower PEGDGE MW ( $\left.z=600 \mathrm{~g} \mathrm{~mol}^{-1}\right)$ with a constant NG and PEGDGE concentrations, the gel possessed a larger amount of epoxide reacting groups; therefore, the crosslink density and modulus were highest. However, the fracture strain decreased with low PEGDGE MW (Figure 3B) because the elastically effective chains were relatively short. On the contrary, the NG-9-PEG-45-6000 gel comprised polymer chains with more mobility and better

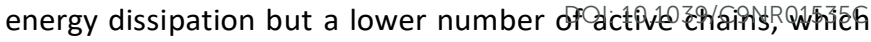
can be subjected to significantly higher compressive stress and strain. The low elastic modulus is due to the long, flexible PEGDGE6000 chains, as would be expected from classic rubber elastic theory. ${ }^{52}$

It is evident that PEGDGE6000 enhanced the ductility of the gels. To further investigate the effect of PEGDGE6000 concentration on the gel mechanical properties, a series of NG$x$-PEG-y-6000 gels $(x=9.1-13.3 w t . \%$ and $y=45.5-20.0$ wt.\%, respectively) were studied where the initial NG mass/concentration was fixed. Network formation occurred after $12 \mathrm{~h}$ at $37^{\circ} \mathrm{C}$. By adding in different PEGDGE6000 masses, the final PEGDGE6000 concentration $(y)$ varied and ultimately changed the final NG concentration $(x)$. Figure $3 C$ and Figure 3D show the mechanical properties with different $y$ values. As seen in Figure 3C, hydrogels prepared with lower $y$ exhibited remarkable ductility. The compressive stress, strain and fracture energy density increased when the total PEGDGE6000 concentration decreased from $y=45.5 \mathrm{wt}$.\% to $20.0 \mathrm{wt}$.\%. The fracture energy density is a measure of the toughness of the hydrogels under compression. ${ }^{85}$ Notably, both NG-13-PEG-296000 and NG-13-PEG-20-6000 showed high compressive stress of 23.4 MPa and 24.2 MPa at high compressive strains of $94 \%$ and $98 \%$, respectively. Meanwhile, NG-13-PEG-20-6000 exhibited a fracture energy density of greater than $1.88 \mathrm{MJm}^{-3}$ because it did not fracture at the maximum strain, which is comparable to values of other free-radical double and triple networks. ${ }^{46,85}$ These results suggested that this is the hydrogel with optimum mechanical properties within the series.

Increasing y above $29.0 \mathrm{wt}$.\% increases the number of free and singly grafted polymers. This results in lower crosslink density between the epoxide and carboxylic acid groups due to greater shielding/entrapment by free and singly grafted polymers. Therefore, the fracture energy density and compressive stress decreased significantly. Since all effective crosslinked PEGDGE6000 chains possess the same length, the fracture strains could remain the same. As seen in Figure 3D, the fracture strain showed only a slight decrease at $y>29 \mathrm{wt} . \%$. On the other hand, the modulus values remained similar at most $y$ values, except for a lower modulus at $y=20 \mathrm{wt} . \%$. This unexpected result can be attributed to the contribution of a saturated population of free and singly grafted PEGDGE6000 when $y \geq 29 w t . \%$. The maxima of all graphs in Figure 3D suggested that for $y<29 w t . \%$ more efficient crosslinking with less singly grafted and free polymers increased the compressive stress and toughness.

To the best of our knowledge, most of the prior studies on PEG-based hydrogels only focused on the cell studies rather their mechanical properties ${ }^{10-34}$ and only a few papers studied their rheological properties. ${ }^{17}, 25,29,32$ Some reports have been published on the syntheses of higher mechanical strength PEGbased hydrogels that contain compression data, ${ }^{23}, 52,86$ even though such properties are crucial to particular applications such as replacements for degenerated articular cartilages and intervertebral discs. Compared to DN hydrogels of high mechanical strength, our PEG-based nanocomposite hydrogel 
exhibited comparably high compressive stress values (Table S1). $44,46,52,61,63,65,71,72$ To further demonstrate their excellent reversible compressibility, a car crush experiment was carried out where the NG-13-PEG-20-6000 gel was run over by a rear tyre of a 1.0 tonne vehicle (Figure S4 and Video S1, ESI + ). As mentioned earlier, the NG-13-PEG-20-6000 gel possessed a very high compressive stress and strain, it remained intact after compression crush by the rear tyre of a moving car highlighting the potential application of these gels for load bearing applications.

To investigate the influence of the NG concentration and their nanostructure on the gels, a series of the NG-x-PEG-206000 gels were studied $(x=6.7-15.3$ wt.\%, Supporting Information, Table S1). Their compressive stress-strain curves, their relevant fracture energy density, modulus and compressive strain versus the final NG concentration $(x)$ were shown in Figure 4A (also Figure S5A, ESI + ). When $x=6.7$ and $10.0 \mathrm{wt} . \%$, the modulus, compressive stress and fracture energy density were low. However, a small increase in $\mathrm{x}$ (from 10.0 to $13.3 \mathrm{wt} . \%$ ) resulted in a striking enhancement of 2.5 times for modulus and 24 times for fracture energy density. The improvement of compressive strength, fracture energy density and modulus are strongly dependent on $\mathrm{x}$. It can be attributed to the fact that when $x$ is low, the NG particles are further apart making it more difficult for the PEGDGE chains to be linked by the NGs, leading to a lower crosslink density. Furthermore, only a slight increase was observed in the fracture strain with increasing NG concentrations since all effective crosslinked PEGDGE6000 chains possess the same length. Nevertheless, with a maxima in all 3 plots in Figure 4A, the NG-13-PEG-206000 gel exhibited the best mechanical properties and is the optimum composition for hydrogel preparation. This can be attributed to an optimal crosslinking density between the NGs and PEGDGE at concentration $x=13 w t . \%$ and $y=20 w t . \%$.
(A)

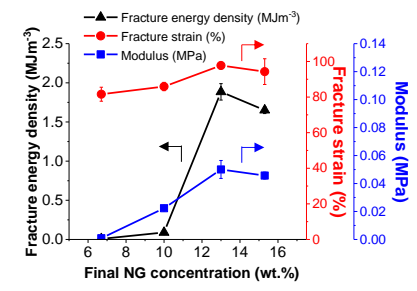

(B)

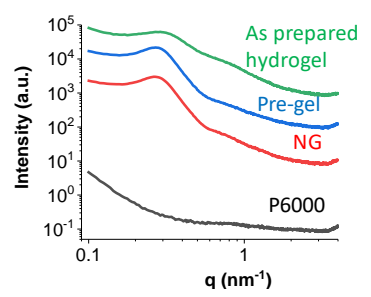

Figure 4. (A) Variation of fracture energy density, strain-at-break and modulus versus final NG concentration for the NG-x-PEG-20-6000 gels with different final NG concentration ratio ( $x=6.7-15.3$ wt.\%). (B) SAXS data for the NG-13-PEG-20-6000 gel as well as data for the initial PEGDGE, NG and the mixture (pre-gel) prior to gel formation.

Scanning electron microscopy (SEM) was used to probe the morphology of freeze-dried NG-13-PEG-20-6000 (Figure S5B, ESIT) and NG particles can be seen closely packing. Small angle $\mathrm{x}$-ray scattering (SAXS) was used to provide more quantitative structural information about the gels (Figure 4B). The presence of a structure factor peak for the concentrated dispersion of NG $(x=20$ wt.\%) demonstrates a highly interacting nature of the particles. ${ }^{87}$ The peak position did not change significantly by the addition of $\mathrm{P} 6000$ (the pre-gel mixture), meaning that the particle-particle separation $(D)$ was minimally perturbed by added PEGDGE (see Figure 4B and Supporteng39hformpationf, Table S2). Using the scattering vector $(q)$ position of the structure factor peak maximum, the average centre-to-centre distance $(D)$ between NG particles was estimated through the relationship $D=2 \pi / q_{\max }$. Peak fittings were performed to determine the $q$-value of peak maxima $\left(q_{\max }\right)$ for all patterns (Figure S6, ESIt). From these fittings, the average distance between NGs in the pre-gel mixture was found to be $21.2 \mathrm{~nm}$, which decreased to $19.5 \mathrm{~nm}$ upon crosslinking (see Table S2, $\mathrm{ESI}+$ ). Furthermore, the appearance of a second order peak (at $q \sim 0.8 \mathrm{~nm}^{-1}$ ) after crosslinking indicates that correlation between NG particles increased and the crosslinked NGs are better ordered in the nanocomposite gel. ${ }^{87}$

One of the important criteria of hydrogels with potential applications as replacements for intervertebral disc or articular cartilage is their repetitive load-bearing ability. The NG-13-PEG20-6000 gels showed excellent recovery after each load cycle for 20 cycles of loading-unloading (see Figure 5A and Figure S7, $\mathrm{ESI}+$ ). The hydrogels were subjected to a maximum compressive stress of $0.93 \mathrm{MPa}$. The maximum compressive strain was more than $70 \%$ and showed little creep. Very low hysteresis was observed as seen in Figure 5A. The dissipated energy calculated from the area of the hysteresis loops decreased slightly at first and then approached constant values (Figure 5B). The gels also showed little residual strain and were highly resilient. Although most chemically crosslinked hydrogels often soften and lack of the ability to recover, 88 our gels are resilient and showed recoverable dissipation energy.
(A)

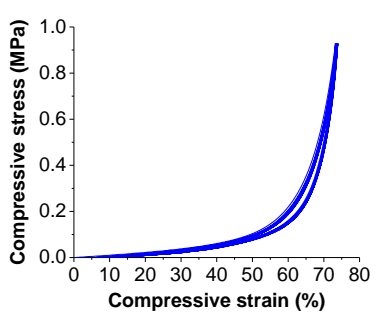

(C)

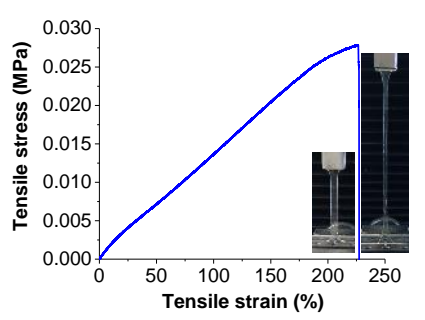

(B)

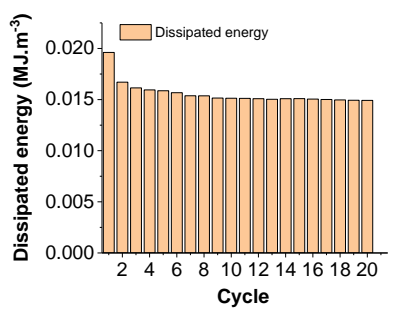

(D)

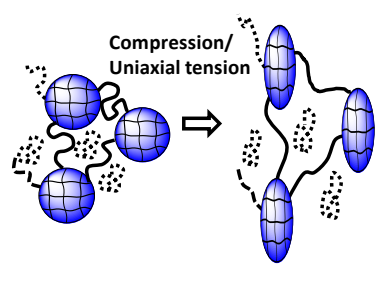

Figure 5. (A) Repetitive loading and unloading compressive data (20 cycles) for the NG13-PEG-20-6000 gel. (B) The corresponding dissipated energy of each cycle. (C) Tensile stress-strain data for the NG-13-PEG-20-6000 gel. The inset photographs show the gel being stretched. (D) Cartoon depicting the proposed changes in gel structure during deformation.

The NG-13-PEG-20-6000 gel also showed a good fracture tensile strain of $230 \%$ (see Figure $5 \mathrm{C}$ ). The efficient energydissipation mechanism to toughen the gels when the network is 
subjected to uniaxial tension/compression may involve the deformation of crosslinked PEGDGE6000 chains between neighbouring NG particles and stretching/compressing of the individual NG themselves, as depicted in Figure 6D. It is proposed that upon stretching the gel the PEGDGE6000 chain effectively absorbed the crack energy by changing their conformation to adapt and withstand the external force, followed by the ultimate fracture at the crosslink points. The reinforcing particles deform also contributing to energy dissipation. ${ }^{56,73,77}$ Therefore, more efficient crosslinking, less free or singly grafted polymers and the nanostructure correlation of the NG particle lead to better energy dissipation. The high mechanical strengths and the load-bearing ability of our gels are comparable to those of hybrid and microspherestructured DN hydrogels reported previously; 65, 72, 86, 89 however, more importantly our gels are prepared in a single step and contain a covalently crosslinked PEG-based network.

$\mathrm{A} \mathrm{pH}$-dependent swelling in $\mathrm{pH}$ range from $5.8-9.8$ of the NG-13-PEG-20-6000 gel was studied and shown in Figure 6A and Figure S8, ESI+. At most pH conditions, equilibrium swelling was reached after 1 day. The gels maintained their self-supporting physical forms even after soaking for 90 days in PBS indicating effective and stable covalent crosslinking. Environmentally responsive hydrogels, in general, can be used for many applications. In particular, pH-dependent swelling hydrogels can be used in applications including drug delivery ${ }^{90}$, gel actuators $^{91}$ and sensors ${ }^{92}$. In addition, for the potential application as replacements for intervertebral discs or cartilages, our group has demonstrated that $\mathrm{pH}$-responsive hydrogels restore the mechanical properties of degenerated intervertebral discs. ${ }^{42,}, 93$

(A)

(C)
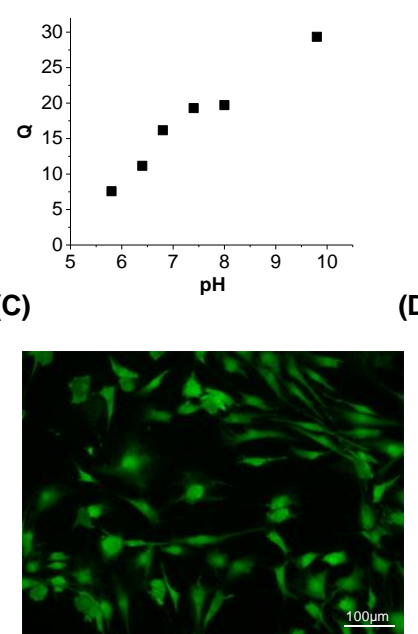

(D)

(B)

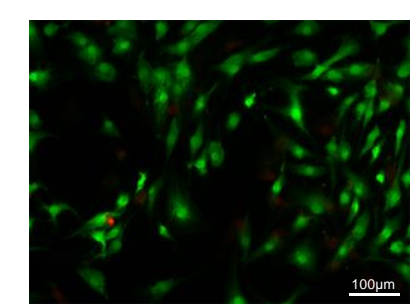

Figure 6. The properties of NG-13-PEG-20-6000 gel: (A) Equilibrium swelling ratio in different $\mathrm{pH}$ conditions. (B) Effect of swelling on the mechanical properties. Cell challenge data are shown in (C) and (D) for a control and a gel, respectively, after 8 days.

At equilibrium swelling, the volume swelling ratio increased from 7.6 to 19.3 and up to 29.3 for the $\mathrm{pH}$ conditions of $5.8,7.4$ and 9.8 respectively. SAXS data demonstrated that swelling led to a shift in $q_{\max }$ value of the structure factor peak towards lower values (Figure S9, ESI ${ }^{+}$), indicating a more significant swelling under basic conditions as carboxylate groyps in the NG

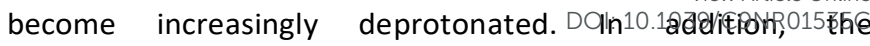
disappearance of the higher order peak upon swelling indicates that the correlation between the NGs decreased. As expected, the significant swelling in PBS buffer at $\mathrm{pH} 7.4$ led to decrease of the hydrogel's excellent ductility. Nevertheless, the gel still exhibited a compressive stress of $0.23 \mathrm{MPa}$ (Figure 6B). The decreased performance of the mechanical properties coincides with loss of the well-ordered NG nanostructure upon $\mathrm{pH}$ triggered swelling of NGs and the extension of hydrophilic PEGDGE6000 in water.

Many reports on hydrogels with excellent mechanical strength are mostly based on the $\mathrm{DN}, 46$ hybrid $\mathrm{DN}^{71,72}$ and micro/nano particle reinforced DN. ${ }^{52,61,63,65}$ In most cases, crosslinking via free-radical polymerisation often involves the use of toxic acrylamide as a starting material. As a result, their potential biomaterial applications may be limited. Here, the cytotoxicity of the NG-13-PEG-20-6000 gel was tested using human nucleus pulposus (NP) cells. The NP cells were cultured with toroid shaped gels. The results suggested that all cells were viable after 8 days of culture time (Figure 6C and 6D), and more data concerning the cytotoxicity experiments are shown in Figure S10, ESIt. The overall data support the potential for our nanocomposite hydrogel as load bearing biomaterial implants.

\section{Conclusion}

In this work, we developed a new strategy to prepare highly compressive and stretchable PEG-based hydrogels without free-radical chemistry using $\mathrm{pH}$-responsive NGs. Different MW PEGDGE was able to be used to tune the mechanical properties of the hydrogels. Oscillatory shear rheology and uniaxial compressive tests confirmed that using a commercially available PEGDGE6000 as the matrix enhanced the properties of the gels. Densely packed nanostructures of the gels were confirmed by SEM and SAXS analysis. For a series of the NG-XPEG-20-6000 gels with different final NG concentration ratios ( $x$ $=6.7-15.3$ wt.\%), the NG-13-PEG-20-6000 exhibited high compressive stress of $24.2 \mathrm{MPa}$ at compressive strain $96 \%$ and a good fracture tensile strain of $230 \%$. They also exhibited fracture energy density as high as $1.88 \mathrm{MJ} \mathrm{m}^{-3}$, which is comparable to values of other free-radical double and triple networks. They survived crush run over by a rear tyre of a 1.0 tonne car highlighting the potential application of these gels for load bearing applications. Their repetitive load-bearing ability suggested that our gels are highly resilient. The NG-13-PEG-206000 gel demonstrated a $\mathrm{pH}$-dependent swelling in $\mathrm{pH}$ condition range from $5.8-9.8$. The cytotoxicity data for the NG13-PEG-20-6000 gel suggested that all human nucleus pulposus cells were viable after 8 days of culture time. The overall results highlight the outstanding mechanical properties and low cytotoxicity of our gels, which showcase their potential as injectable/implant replacements for intervertebral discs or articular cartilages in the in vivo loading state where swelling is strictly limited. Thus, PEGDGE with higher MW could be 
synthesised as potential candidates for even better 23 . compression and tensile properties.

\section{Conflicts of interest}

There are no conflicts to declare.

\section{Acknowledgements}

This work was supported by a 5 year EPSRC Established Career Fellowship awarded to BRS (M002020/1).

\section{References}

1. B. Jeong, Y. H. Bae and S. W. Kim, J. Controlled Release, 2000, 63, 155-163.

2. J. L. Drury and D. J. Mooney, Biomaterials, 2003, 24, 43374351

3. J. B. Leach and C. E. Schmidt, Biomaterials, 2005, 26, 125135

4. N. A. Peppas, H. J. Z., K. A. and L. R., Adv. Mater., 2006, 18 $1345-1360$.

5. A. S. Hoffman, Adv. Drug Delivery Rev., 2012, 64, 18-23.

6. C.-C. Lin and K. S. Anseth, Pharm. Res., 2009, 26, 631-643.

$7 . \quad J . Z h u$, Biomaterials, 2010, 31, 4639-4656.

8. J. A. Hubbell, Curr. Opin. Solid State Mater. Sci., 1998, 3, 246-251.

9. E. Bakaic, N. M. B. Smeets and T. Hoare, RSC Adv., 2015, 5, 35469-35486.

10

N. A. Peppas, K. B. Keys, M. Torres-Lugo and A. M. Lowman J. Controlled Release, 1999, 62, 81-87.

11. J. Elisseeff, K. Anseth, D. Sims, W. McIntosh, M. Randolph and R. Langer, Proc. Natl. Acad. Sci. U. S. A., 1999, 96, 31043107.

12. A. N. Buxton, J. Zhu, R. Marchant, J. L. West, J. U. Yoo and B. Johnstone, Tissue Eng., 2007, 13, 2549-2560.

13. M. S. Hahn, M. K. McHale, E. Wang, R. H. Schmedlen and J. L. West, Ann. Biomed. Eng., 2007, 35, 190-200.

14. J. Elisseeff, W. McIntosh, K. Anseth, S. Riley, P. Ragan and R. Langer, J. Biomed. Mater. Res., 2000, 51, 164-171.

15. J. S. Miller, C. J. Shen, W. R. Legant, J. D. Baranski, B. L. Blakely and C. S. Chen, Biomaterials, 2010, 31, 3736-3743.

16. A. Metters and J. Hubbell, Biomacromolecules, 2005, 6, 290-301.

R. Jin, L. S. Moreira Teixeira, A. Krouwels, P. J. Dijkstra, C. A. van Blitterswijk, M. Karperien and J. Feijen, Acta Biomater. 2010, 6, 1968-1977.

18

L. S. Qiong, Y. Chuan, H. Yuan, D. Xin, L. Yan, F. W. Min, H. J. L. and Y. Yi-Yan, Adv. Mater., 2012, 24, 6484-6489.

19. M. Malkoch, R. Vestberg, N. Gupta, L. Mespouille, P. Dubois, A. F. Mason, J. L. Hedrick, Q. Liao, C. W. Frank, K. Kingsbury and C. J. Hawker, Chem. Commun., 2006, 0, 2774-2776.

20. C.-C. Lin, A. Raza and H. Shih, Biomaterials, 2011, 32, 9685 9695.

21. L. J. Macdougall, M. M. Pérez-Madrigal, M. C. Arno and A. P. Dove, Biomacromolecules, 2018, 19, 1378-1388.

22. Q. Lu, Y. B. He, Q. Yu, B. Li, Y. V. Kaneti, Y. Yao, F. Kang and Q. H. Yang, Adv. Mater., 2017, 29, 1604460.
N. Mac Kenna, P. Calvert, A. Morrin, G. G. Wallace and S. E. Moulton, J. Mater. Chem. B, 2015, [3,2530025379NR01535C

24. Y. Privar, I. Malakhova, A. Pestov, A. Fedorets, Y. Azarova, S. Schwarz and S. Bratskaya, Chem. Eng. J., 2018, 334, 1392-1398.

25. H.-D. Hwang, H.-J. Cho, P. Balakrishnan, C.-W. Chung, I.-S Yoon, Y.-K. Oh, Y. Byun and D.-D. Kim, Colloids Surf., $B$ 2012, 91, 106-113.

26. T. Segura, B. C. Anderson, P. H. Chung, R. E. Webber, K. R. Shull and L. D. Shea, Biomaterials, 2005, 26, 359-371.

$27 . \quad$ T. Segura, P. H. Chung and L. D. Shea, Biomaterials, 2005 26, 1575-1584.

28. M. Nishida, Y. Uraki and Y. Sano, Bioresour. Technol., 2003, 88, 81-83.

29. R. Barbucci, D. Pasqui, R. Favaloro and G. Panariello, Carbohydr. Res., 2008, 343, 3058-3065.

M. N. Collins and C. Birkinshaw, J. Appl. Polym. Sci., 2008 109, 923-931.

T. J. Ohara, R. Rajagopalan and A. Heller, Anal. Chem., 1994, 66, 2451-2457.

L. Passauer, K. Fischer and F. Liebner, Journal, 2011, 65 , 309.

L. Passauer, M. Struch, S. Schuldt, J. Appelt, Y. Schneider D. Jaros and H. Rohm, ACS Appl. Mater. Interfaces., 2012, 4, 5852-5862.

34

T. Yuan, J. Meng, T. Hao, Z. Wang and Y. Zhang, ACS Appl. Mater. Interfaces., 2015, 7, 14896-14904.

L. Shechter and J. Wynstra, Ind. Eng. Chem., 1956, 48, 8693.

H. Kakiuchi and Y. Tanaka, J. Org. Chem., 1966, 31, 15591564.

F. B. Alvey, J. Polym. Sci., Part A: Polym. Chem., 1969, 7 2117-2124.

38. A. V. Reis, A. R. Fajardo, I. T. A. Schuquel, M. R. Guilherme, G. J. Vidotti, A. F. Rubira and E. C. Muniz, J. Org. Chem., 2009, 74, 3750-3757.

39. R. Liu, A. H. Milani, T. J. Freemont and B. R. Saunders, Soft Matter, 2011, 7, 4696-4704.

R. Liu, A. H. Milani, J. M. Saunders, T. J. Freemont and B. R. Saunders, Soft Matter, 2011, 7, 9297-9306.

A. H. Milani, J. Bramhill, A. J. Freemont and B. R. Saunders, Soft Matter, 2015, 11, 2586-2595.

42. A. H. Milani, L. A. Fielding, P. Greensmith, B. R. Saunders, D. J. Adlam, A. J. Freemont, J. A. Hoyland, N. W. Hodson, M. A. Elsawy, A. F. Miller, L. P. D. Ratcliffe, O. O. Mykhaylyk and S. P. Armes, Chem. Mater., 2017, 29, 3100-3110.

43. A. H. Milani, J. M. Saunders, N. T. Nguyen, L. P. D. Ratcliffe, D. J. Adlam, A. J. Freemont, J. A. Hoyland, S. P. Armes and B. R. Saunders, Soft Matter, 2017, 13, 1554-1560.

T. Lane, J. L. Holloway, A. H. Milani, J. M. Saunders, A. J. Freemont and B. R. Saunders, Soft Matter, 2013, 9, 79347941.

45. N. L. Nerurkar, D. M. Elliott and R. L. Mauck, J. Biomech. 2010, 43, 1017-1030.

46. J. P. Gong, Y. Katsuyama, T. Kurokawa and Y. Osada, Adv Mater., 2003, 15, 1155-1158.

47. K. Wei, X. Chen, R. Li, Q. Feng and L. Bian, Chem. Mater., 2017, 29, 8604-8610.

48. X. Chen, C. Dong, K. Wei, Y. Yao, Q. Feng, K. Zhang, F. Han, A. F.-T. Mak, B. Li and L. Bian, NPG Asia Materials, 2018, 10 788-799. 
49.

Q. Feng, K. Wei, K. Zhang, B. Yang, F. Tian, G. Wang and L. Bian, Npg Asia Materials, 2018, 10, e455.

50. K. Haraguchi and T. Takehisa, Adv. Mater., 2002, 14, 11201124.

51. T. Serizawa, K. Wakita and M. Akashi, Macromolecules, 2002, 35, 10-12.

52. T. Huang, H. G. Xu, K. X. Jiao, L. P. Zhu, H. R. Brown and H. L. Wang, Adv. Mater., 2007, 19, 1622-1626.

53. S. M. Kyoon, S. G. M., S. S. Ryon, K. S. I. and K. S. Jeong, $A d v$. Mater., 2009, 21, 1712-1715.

54. W.-C. Lin, W. Fan, A. Marcellan, D. Hourdet and C. Creton, Macromolecules, 2010, 43, 2554-2563.

55. J. Hu, K. Hiwatashi, T. Kurokawa, S. M. Liang, Z. L. Wu and J. P. Gong, Macromolecules, 2011, 44, 7775-7781.

56. J. Hu, T. Kurokawa, T. Nakajima, Z. L. Wu, S. M. Liang and J. P. Gong, Macromolecules, 2014, 47, 3587-3594.

57. J. Meid, F. Dierkes, J. Cui, R. Messing, A. J. Crosby, A. Schmidt and W. Richtering, Soft Matter, 2012, 8, 42544263.

58. J. Liu, C. Chen, C. He, J. Zhao, X. Yang and H. Wang, ACS Nano, 2012, 6, 8194-8202.

59. X. Yang, E. Bakaic, T. Hoare and E. D. Cranston, Biomacromolecules, 2013, 14, 4447-4455.

60. L.-W. Xia, R. Xie, X.-J. Ju, W. Wang, Q. Chen and L.-Y. Chu, Nat. Commun., 2013, 4, 2226.

61. Y.-n. Sun, G.-r. Gao, G.-I. Du, Y.-j. Cheng and J. Fu, ACS Macro Lett., 2014, 3, 496-500.

62. H. Shin, B. D. Olsen and A. Khademhosseini, J. Mater. Chem $B, 2014,2$, 2508-2516.

63. G. Gao, G. Du, Y. Cheng and J. Fu, J. Mater. Chem. B, 2014, 2, 1539-1548.

64. H. K. Heris, J. Daoud, S. Sheibani, H. Vali, M. Tabrizian and L. Mongeau, Adv. Healthcare Mater., 2016, 5, 255-265.

65. D. Jiangjiang, L. Xichao, G. Jinhua, Z. Kunkun and Z. Lina, Adv. Mater., 2016, 28, 8037-8044.

66. H. Jiang, G. Zhang, F. Li, Y. Zhang, Y. Lei, Y. Xia, X. Jin, X. Feng and H. Li, Nanoscale, 2017, 9, 15470-15476.

67. B. B. Mendes, M. Gómez-Florit, R. A. Pires, R. M. A. Domingues, R. L. Reis and M. E. Gomes, Nanoscale, 2018, 10, 17388-17401.

68. S. Dai, X. Zhou, S. Wang, J. Ding and N. Yuan, Nanoscale, 2018, 10, 19360-19366.

69. H. Xu, Y. Lv, D. Qiu, Y. Zhou, H. Zeng and Y. Chu, Nanoscale, 2019, DOI: 10.1039/C8NR08589G.

70. J. P. Gong and Y. Osada, in High Solid Dispersions, ed. M. Cloitre, Springer, Berlin, Heidelberg, 2010, vol. 236, Advances in Polymer Science, pp. 203-246.

71. C. Jinfeng, L. Jiahong, C. Yumei, Z. Lina and Z. Jinping, $A d v$. Funct. Mater., 2018, 28, 1800739.

72. Y. Yanyu, W. Xing, Y. Fei, W. Luning and W. Decheng, Adv. Mater., 2018, 30, 1707071.

73. J. Hu, T. Kurokawa, T. Nakajima, T. L. Sun, T. Suekama, Z. L. Wu, S. M. Liang and J. P. Gong, Macromolecules, 2012, 45, 9445-9451.

74. Y. Nomura, R. Hörlein, P. Tzallas, B. Dromey, S. Rykovanov, Z. Major, J. Osterhoff, S. Karsch, L. Veisz, M. Zepf, D. Charalambidis, F. Krausz and G. D. Tsakiris, Nat. Phys., 2008, 5, 124.

75. R. Delhez, T. H. de Keijser and E. J. Mittemeijer, Fresenius' Zeitschrift für analytische Chemie, 1982, 312, 1-16.

76. R. G. Kirste and R. C. Oberthuer, Small Angle Scattering, Academic Press, London, 1982.
77.

J. Hu, T. Kurokawa, K. Hiwatashi, T. Nakajima, Z L. Wu S. M. Liang and J. P. Gong, Macromotecules, 22012,45, 523185228.

78. T. Vidil and F. Tournilhac, Macromolecules, 2013, 46, 92409248.

79. B. R. Saunders, H. M. Crowther and B. Vincent, Macromolecules, 1997, 30, 482-487.

80. G. M. Kavanagh and S. B. Ross-Murphy, Prog. Polym. Sci., 1998, 23, 533-562.

81. D. E. Clarke, E. T. Pashuck, S. Bertazzo, J. V. M. Weaver and M. M. Stevens, J. Am. Chem. Soc., 2017, 139, 7250-7255.

82. S. Abbas, Z. Judit and P. Donald, Phys. Med. Biol., 2007, 52, 1565.

83. H. Saraf, K. T. Ramesh, A. M. Lennon, A. C. Merkle and J. C. Roberts, J. Biomech., 2007, 40, 1960-1967.

84. D. Kaneko, T. Tada, T. Kurokawa, J. P. Gong and Y. Osada, Adv. Mater., 2005, 17, 535-538.

85. P. A. Panteli and C. S. Patrickios, Macromolecules, 2018, 51, 7533-7545.

86.

V. X. Truong, M. P. Ablett, S. M. Richardson, J. A. Hoyland and A. P. Dove, J. Am. Chem. Soc., 2015, 137, 1618-1622.

87. T. Suteewong, H. Sai, J. Lee, M. Bradbury, T. Hyeon, S. M. Gruner and U. Wiesner, J. Mater. Chem., 2010, 20, 78077814.

88. T. Aida, E. W. Meijer and S. I. Stupp, Science, 2012, 335 813.

89. Q. Chen, L. Zhu, H. Chen, H. Yan, L. Huang, J. Yang and J. Zheng, Adv. Funct. Mater., 2015, 25, 1598-1607.

90. Y. Qiu and K. Park, Adv. Drug Delivery Rev., 2001, 53, 321339.

91. K.-F. Arndt, D. Kuckling and A. Richter, Polym. Adv. Technol., 2000, 11, 496-505.

92. A. Richter, A. Bund, M. Keller and K.-F. Arndt, Sensors Actuators B: Chem., 2004, 99, 579-585.

93. A. H. Milani, A. J. Freemont, J. A. Hoyland, D. J. Adlam and B. R. Saunders, Biomacromolecules, 2012, 13, 2793-2801. 


\section{Nanoscale}

\section{ARTICLE}

\section{The table of contents entry}

Title: Highly Compressive and Stretchable Poly(Ethylene Glycol) based Hydrogels Synthesized using pHResponsive Nanogels without Free-Radical Chemistry.

Nam T. Nguyen ${ }^{a, *}$, Amir H. Milani ${ }^{a}$, James Jennings ${ }^{b}$, Daman J. Adlam ${ }^{c}$, Anthony J. Freemont ${ }^{c}$, Judith A. Hoyland ${ }^{c, d}$ and Brian R. Saunders. ${ }^{a}$

A new strategy is developed to prepare a novel class of highly compressive and stretchable PEG-based hydrogels using $\mathrm{pH}$-responsive nanogels.

ToC figure:

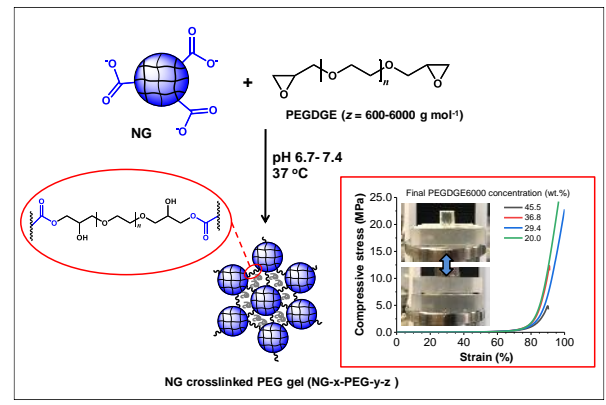

